\title{
Solothurn: erster Kanton mit einheitlichem Betreuungsplan für Schwerkranke
}

\author{
Der Verein «palliative so» führt gemeinsam mit der Gesellschaft der Ärztinnen \\ und Ärzte und der Solothurner Spitäler AG mit Unterstützung des Kantons sowie \\ der Ärztekasse den neu entwickelten Betreuungsplan für Palliative-Care-Patienten \\ ein. Dank dieses Instruments soll die Behandlungskette bei unheilbar- und schwer- \\ kranken Menschen nicht reissen.
}

Christoph Cina

Präsident palliative so
Palliative Care bietet eine bestmögliche Lebensqualität bis zum natürlichen Tod. Dazu gehört, dass Schmerzen und andere Leiden gelindert werden, und dass Betroffene und Angehörige individuell begleitet werden. Es bedeutet auch, dass ein stabiles Netzwerk von Fachpersonen, Freiwilligen und Angehörigen eine nahtlose Versorgung am Lebensende gewährleistet. Patientinnen und Patienten mit schweren, unheilbaren Krankheiten am Lebensende erleben jedoch oft mehrere Übergänge von einer Institution in die andere und auch wieder zurück nach Hause. Dabei können Lücken in der Behandlungskette entstehen. Um dies zu vermeiden, hat «Palliative Solothurn» einen Betreuungsplan entwickelt.

\section{Elektronische Aufbereitung der Krankengeschichte am Lebensende}

Die Informationsmenge und Komplexität der Krankengeschichte von Menschen in ihrer letzten Lebensphase kann ohne eine strukturierte elektronische Erfassung in Zukunft nicht mehr bewältigt werden. Der Betreuungsplan des Kantons Solothurn wurde als Basisdokument für die interdisziplinäre Betreuung von Patientinnen und Patienten entwickelt und er basiert auf Konsens. Der Kanton Solothurn ist somit schweizweit der erste, der ein einheitliches Arbeitsinstrument bei Palliative-Care-Patientinnen und -Patienten einsetzt. verändert werden. Spitäler und Hausärzte des Kantons Solothurns haben in einer Testphase mit dem Betreuungsplan und dem Online-Patientendossier gearbeitet. Damit erproben sie ein zukunftsweisendes Schnittstellenmanagement. Das Online-Patientendossier ermöglicht eine elektronische Führung der Krankengeschichte, bei der unter anderem Pflegeberichte, ein Medikamentenplan und ein Kommunikationstool integriert sind. Der Betreuungsplan kann als Basisdokument unabhängig vom Online-Patientendossier ausgedruckt oder in eine Praxis- bzw. SpitalKrankengeschichte integriert werden.

\section{Vorteile des Online-Betreuungsplans}

Durch die Erfassung der vorhandenen und vermutlich sich einstellenden Beschwerden wird ein Betreuungs- und Behandlungskonzept in interprofessioneller Zusammenarbeit mit der Patientin, dem Patienten und allen Betroffenen erarbeitet. Der Medikamentenplan soll regelmässig überwacht und angepasst werden. Unnötige Medikamente werden abgesetzt. Fragen wie die Gabe von Antibiotika, Reanimation, künstliche Beatmung, Transfusion usw. werden vorausschauend vom Betreuungsteam unter Einbezug der Patientin, des Patienten behandelt.

Unnötige Spitalaufenthalte oder erneute Spitalaufenthalte und Doppelspurigkeiten können vermieden werden, was in gewissen Fällen dazu beitragen kann, Kosten und Ressourcen zu sparen.

\section{Der Betreuungsplan wurde als Basisdokument für die interdisziplinäre Betreuung von Patienten entwickelt und er basiert auf Konsens.}

Korrespondenz: Dr. med. Christoph Cina Präsident palliative so Hauptstrasse 16 CH-3254 Messen Tel. 0317655226 christoph.cina[at]hin.ch
Der Betreuungsplan wird in einem elektronischen Patientendossier abgelegt. Die Datenhoheit bleibt selbstverständlich bei den Patienten. Sie bestimmen, wer Einsicht in den Betreuungsplan nehmen darf. Entscheidungen können jederzeit widerrufen oder

\author{
Verschiedene Situationen - \\ ein Betreuungsplan \\ Im Betreuungsplan werden wichtige Fragen am \\ Lebensende angesprochen und geklärt, der Patien- \\ tenwille wird respektiert. Drei Beispiele zeigen, dass
}




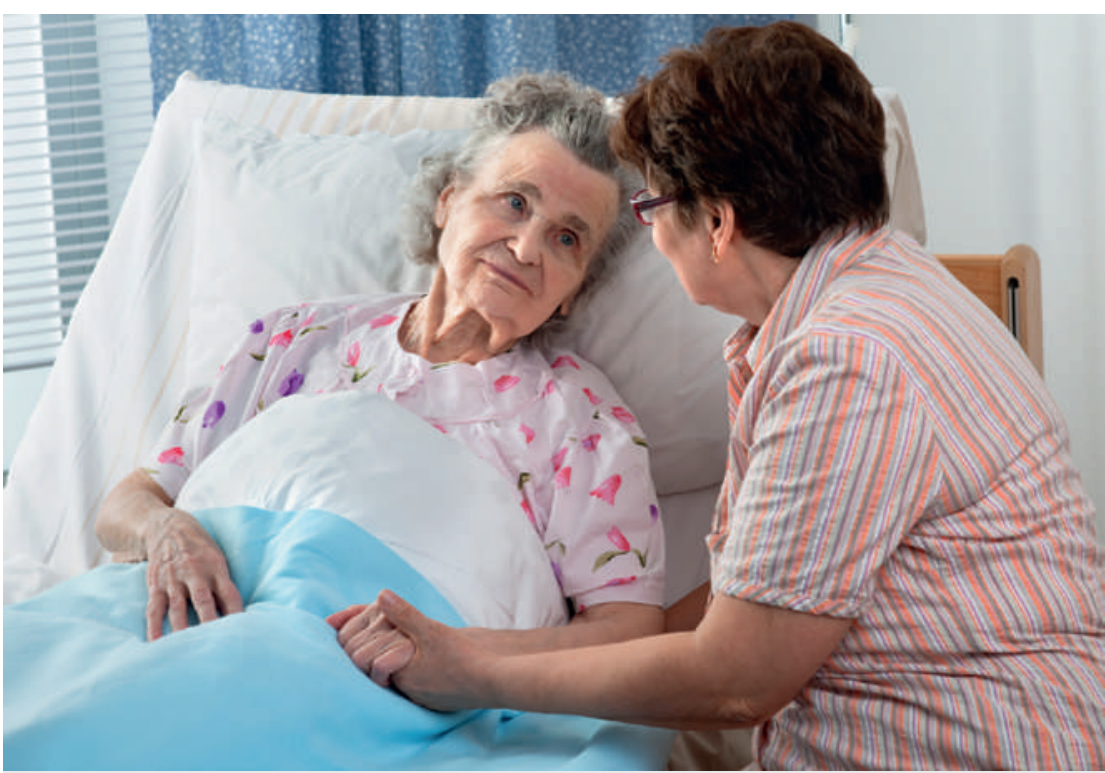

Der Betreuungsplan gewährleistet, dass ein stabiles Netzwerk von Fachpersonen, Freiwilligen und Angehörigen eine nahtlose Versorgung am Lebensende gewährleistet. rung. Sie wird von verschiedenen Fachspezialisten intensiv betreut. Ihre terminale Niereninsuffizienz mit Dialyse und ihr Diabetes mellitus erfordert wiederholte Spitalaufenthalte. Während ihrer letzten Hospitalisation wurde die Diagnose eines nicht kurativen Krebsleidens gestellt. Frau C. wünscht, ihre letzte Lebensphase daheim zu verbringen. Nach Rücksprache mit dem Hausarzt wird der Betreuungsplan im Spital ausgefüllt und die Patientin wird nach Hause entlassen. Der ausgefüllte Betreuungsplan bildet die Basis für die weitere Betreuung zu Hause. Frau C. stirbt daheim in Anwesenheit ihrer Angehörigen.

Die zunehmend pflegebedürftige, demente 90-jährige Frau G. tritt ins Altersheim ein. Zusammen mit der Patientin, ihrer Tochter, dem Hausarzt und der Pflege wird der Betreuungsplan ausgefüllt. Damit wird gewährleistet, dass der Wille der Patientin, die ihre Tochter als Bezugsperson bezeichnet, respektiert wird und die zu erwartenden Symptome und deren Behandlung vorausschauend diskutiert werden. Durch die Klarheit der Angaben werden unnötige Hospitalisationen vermieden und dem Notfallarzt eine Entscheidungshilfe gegeben.

der Betreuungsplan in ganz verschiedenen Situationen zum Einsatz kommen kann.

Der 60-jährige Herr B. meldet sich wegen Schluckbeschwerden bei seinem Hausarzt. Die medizinischen Abklärungen ergeben die Diagnose eines Adenokarzinoms im gastro-oesophagealen Übergang. Herr B. wird operiert, anschliessend radio- und chemotherapiert. Nach einer praktisch beschwerde-

\section{Selbstbestimmung am Lebensende}

Der Betreuungsplan ist ein Arbeitsinstrument, das hilft, Wünsche von Patientinnen und Patienten direkt anzusprechen. Nicht selten werden in einer kurativ orientierten Medizin schwerkranke Patienten gar nicht oder nur oberflächlich zu ihren Erwartungen gefragt. Deshalb beinhaltet der Betreuungs-

\section{Der Betreuungsplan beinhaltet neben medizinischen auch psychologische, soziale und spirituelle Aspekte.}

freien Phase kommt es zu einem Rezidiv mit erneuten Schluckstörungen und Bauchschmerzen. Gemeinsam mit den Angehörigen wird der Betreuungsplan in der Hausarztpraxis besprochen, schriftlich festgehalten und dem Patienten ausgehändigt. Der Betreuungsplan begleitet den Patienten bei den wiederholten Hospitalisationen. Herr B. verstirbt im Spital in Anwesenheit seiner Angehörigen. Es war sein ausdrücklicher Wille, seine letzten Tage im Spital zu verbringen.

Frau C. ist eine 30-jährige Frau mit einer komplexen Leidensgeschichte mit körperlicher Behinde- plan neben den medizinischen auch psychologische, soziale und spirituelle Aspekte. Er schafft die Voraussetzung für einen respektvollen Umgang, der bei allen Beteiligten wesentlich zur Verbesserung der Lebensqualität am Lebensende beiträgt.

Will man Palliative Care besser im Gesundheitswesen verankern, ist ein gut funktionierendes Schnittstellenmanagement eine notwendige Voraussetzung, was auch im Sinne der Nationalen Strategie Palliative Care 2010-2012 ist. Die Nationale Strategie Palliative Care wird auch nach dem Jahr 2012 weitergeführt. 